\title{
Reproductive Human Cells, Tissues, and Cellular and Tissue-Based Products from Known Donor to Directed Recipient
}

National Cancer Institute

\section{Source}

National Cancer Institute. Reproductive Human Cells, Tissues, and Cellular and TissueBased Products from Known Donor to Directed Recipient. NCI Thesaurus. Code C133315.

Reproductive Human Cells, T issues and T issue based Products (HCT/Ps) donated by a donor to a specific recipient who knows and is known by the recipient before donation. 